\title{
АНАЛИЗ КОРРЕЛЯЦИИ СОДЕРЖАНИЯ ЦИТОКИНОВ ПЕРИФЕРИЧЕСКОЙ КРОВИ С РИСКОМ РАЗВИТИЯ СЕРДЕЧНО-СОСУДИСТЫХ ОСЛОЖНЕНИЙ У БОЛЬНЫХ ЭССЕНЦИАЛЬНОЙ АРТЕРИАЛЬНОЙ ГИПЕРТЕНЗИЕЙ ІІ СТАДИИ
}

\author{
О. А. Радаева ${ }^{1}$, А. С. Симбирцев²
}

Мордовский государственный университет имени Н. П. Огарева, Саранск, Россия

2 Государственный научно-исследовательский институт особо чистых биопрепаратов Федерального медико-биологического агентства, Санкт-Петербург, Россия Эссенциальная артериальная гипертензия (ЭАГ) остается наиболее распространенным модифицируемым фактором риска сердечно-сосудистых заболеваний и смерти. Целью исследования было выявить корреляцию содержания цитокинов (интерлейкинов, молекул адгезии, факторов некроза опухоли, роста и др.) в сыворотке периферической крови и частоты осложнений (инфаркта миокарда, острого нарушения мозгового кровообращения) в последующие 5 лет у больных ЭАГ II стадии. У 200 пациентов с ЭАГ ॥ стадии с длительностью заболевания 10-14 лет, получавших сопоставимую гипотензивную терапию и показавших целевые уровни АД, с помощью ИФА в сыворотке периферической крови определяли содержание 28 цитокинов (IL1 $\beta$, IL1 $\alpha$, IL1ra, IL18, IL18BP, IL37, IL6, slL6r, LIF, sLIFr, IGF-1, IGFBP-1, TNFa, sTNF-RI, sVCAM-1, IL17, IL2, IL4, IL10, TGF- $\beta 1$, IL8, CX3CL1, CXCL10, INFy, M-CSF, IL34, VEGF-A, эритропоэтина) и вазоактивных пептидов (NO, iNOS, eNOS, ADMA, SDMA, Nt-proCNP, Nt-proBNP). B течение последующих 5 лет фиксировали случаи развития осложнений. Ретроспективный анализ показал, что для группы с развитием осложнений в последующие 5 лет характерно предварительное снижение уровней IL 1 ra $(p<0,001)$ и IL10 $(p<0,001)$ на фоне повышения содержания IL $1 \beta$ ( $p<0,001)$, TNFa $(p<0,001)$ и M-CSF ( $>$ < 0,001) в сыворотке крови при сравнении с группой без осложнений. Многофакторный анализ с включением в регрессионную модель Кокса ряда показателей: IL1 > 18,8 пг/мл; IL1ra < 511 пг/мл; IL6 > 23,8 пг/мл; IL10 < 26,3 пг/мл; 389 пг/мл < M-CSF < 453 пг/Mл; ADMA > 0,86 мкMоль/л; общий холестерин > 4,9 ммоль/л; ЛПНП > 3,0 ммоль/л; ЛПВП у мужчин < 1,0 ммоль/л; у женщин < 1,2 ммоль/л выявил независимый характер «влияния» на повышение частоты повреждения органов-мишеней при ЭАГ II стадии следующих показателей: содержания M-CSF в диапазоне 389-453 пг/мл ( $p<0,001)$, а также уровня ЛПНП более 3,0 ммоль/л ( $p<0,01)$. Изменение уровня цитокинов является патогенетически обоснованным предиктором повреждения миокарда и головного мозга у больных ЭАГ II стадии, а определение уровня M-CSF в крови может дополнить классические схемы расчета риска развития сердечно-сосудистых осложнений у данной категории больных.

Ключевые слова: цитокины, эссенциальная артериальная гипертензия, инфаркт миокарда, острое нарушение мозгового кровообращения, колониестимулирующий фактор макросрагов M-CSF

Информация о вкладе авторов: О. А. Радаева — набор группы пациентов, забор материала для исследования, сбор данных, интерпретация результатов исследования, написание и компьютерная подготовка рукописи; А. С. Симбирцев - планирование и разработка методологии исследования, анализ данных, редактирование текста.

Соблюдение этических стандартов: исследование одобрено этическим комитетом МГУ им. Н. П. Огарева (протокол № 12 от 14 декабря 2008); все пациенты подписали добровольное информированное согласие; получение биологического материала для исследования (кровь) производили с учетом положений Хельсинской декларации ВМА (2000) и протокола Конвенции Совета Европы о правах человека и биомедицине (1999).

$\triangle$ Для корреспонденции: Ольга Александровна Радаева

ул. Ульянова, д. 26а, г. Саранск, 430030; vtlbwbyf_79@mail.ru

Статья получена: 16.07.2018 Статья принята к печати: 27.02.2019 Опубликована онлайн: 06.03.2019

DOI: $10.24075 /$ vrgmu.2019.006

\section{INVESTIGATING A CORRELATION BETWEEN THE LEVELS OF PERIPHERAL BLOOD CYTOKINES AND THE RISK FOR CARDIOVASCULAR COMPLICATIONS IN PATIENTS WITH STAGE II ESSENTIAL HYPERTENSION}

Radaeva $\mathrm{OA}^{1} \otimes$, Simbirtsev $\mathrm{AS}^{2}$

National Research Mordovia State University, Saransk, Russia

State Research Institute of Highly Pure Biopreparations, FMBA, St. Petersburg, Russia

Essential hypertension $(\mathrm{EH})$ is one of the most common modifiable risk factors for cardiovascular diseases and death. The aim of this study was to investigate a correlation between the levels of some cytokines (interleukins, adhesion molecules, tumor necrosis and growth factors, etc.) in the peripheral blood of patients with stage $\mathrm{II} \mathrm{EH}$ and the rate of complications (myocardial infarction, acute cerebrovascular events, and transient ischemic attacks) occurring in a 5-year follow-up period. Twenty-eight cytokines were measured using ELISA, including IL1 $\beta$, IL1 $\alpha$, IL1ra, IL18, IL18BP, IL37, IL6, sIL6r, LIF, sLIFr, IGF-1, IGFBP-1, TNF $\alpha$, sTNF-RI, sVCAM-1, IL17, IL2, IL4, IL10, TGF-B1, IL8, CX3CL1, CXCL10, INFy, M-CSF, IL34, VEGF-A, and erythropoietin, and a few vasoactive peptides, including NO, iNOS, eNOS, ADMA, SDMA, Nt-proCNP, and Nt-proBNP, in the peripheral blood samples of 200 patients with stage II EH who had been suffering from this condition for 10 to 14 years and were receiving comparable therapies to bring their blood pressure down. The patients were followed up for 5 years to keep track of complications. The retrospective analysis revealed that the group of patients who developed complications during the 5-year follow-up period exhibited a decline in the levels of IL1ra $(p<0.001)$ and IL10 $(p<0.001)$ and a rise in IL1 $\beta(p<0.001)$, TNF $\alpha(p<0.001)$ and M-CSF $(p<0.001)$ in comparison with the group of those who did not develop any complications. The multivariate Cox regression analysis was applied to the following parameters: IL1 $\beta>18.8 \mathrm{pg} / \mathrm{ml} ; \mathrm{IL} 1 \mathrm{ra}<511 \mathrm{pg} / \mathrm{ml}$; IL6 $>23.8 \mathrm{pg} / \mathrm{ml}$; IL10 < $26.3 \mathrm{pg} / \mathrm{ml} ; 389 \mathrm{pg} / \mathrm{ml}<\mathrm{M}-\mathrm{CSF}<453 \mathrm{pg} / \mathrm{ml}$; ADMA > $0.86 \mu \mathrm{mol} / \mathrm{L} ;$ total cholesterol > $4.9 \mathrm{mmol} / \mathrm{L} ; \mathrm{LDL}>3.0 \mathrm{mmol} / \mathrm{L} ; \mathrm{HDL}$ in men < $1.0 \mathrm{mmol} / \mathrm{L} ; \mathrm{HDL}$ in women $<1.2 \mathrm{mmol} / \mathrm{L}$. The analysis revealed that $\mathrm{M}-\mathrm{CSF}$ in the range from 389 to $453 \mathrm{pg} / \mathrm{ml}(p<0.001)$ and LDL above $3.0 \mathrm{mmol} / \mathrm{L}(p<0.01) \mathrm{correlated}$ with an increase in the risk for end-organ damage in stage $\mathrm{II} \mathrm{EH}$. Changes in the cytokine levels can be regarded as a predictor of myocardial and cerebral damage in patients with stage II EH. Measurement of peripheral blood M-CSF can be included into the classic risk assessment schemes for the cardiovascular complications in the studied cohort of patients.

Keywords: cytokines, essential hypertension, myocardial infarction, stroke, M-CSF

Author contribution: Radaeva OA recruited patients, collected blood samples for the study took medical histories, interpreted the study results, and wrote the manuscript. Simbirtsev AS conceived and planned the study, analyzed the obtained data and revised the manuscript.

Compliance with ethical standards: the study was approved by the Ethics Committee of Ogariov Mordovian State University (Protocol 12 dated December 12 , 2008). All patients gave their informed consent to participate. Blood samples were collected in full compliance with the Declaration of Helsinki (2000) and the Protocol of the Convention on Human Rights and Biomedicine of the Council of Europe (1999).

Correspondence should be addressed: Olga A. Radaeva

Ulianova 26/a, Saransk, 430030; vtlbwbyf_79@mail.ru

Received: 16.07.2018 Accepted: 27.02.2019 Published online: 06.03.2019

DOI: $10.24075 /$ brsmu.2019.006 
Эссенциальная артериальная гипертензия (ЭАГ) является наиболее распространенным модифицируемым фактором риска сердечно-сосудистых заболеваний и смерти. По некоторым оценкам, во всем мире более 1 млрд взрослых страдают ЭАГ, и к 2025 г. эта цифра возрастет до 1,5 млрд [1]. В течение многих лет считалось, что поражение органовмишеней является результатом действия повышенного гидростатического давления на стенки сосудов. В настоящее время в ряде исследований [2-10] изучали цитокиновые звенья развития осложнений ЭАГ (инфаркта миокарда (ИМ), острого нарушения мозгового кровообращения (ОНMK), транзиторного нарушения мозгового кровообращения (ТНМК)), значимых причинных факторов смертности и заболеваемости среди лиц трудоспособного возраста. Не вызывает сомнения, что повреждение органов-мишеней конечная точка хронического воспалительного процесса на фоне повышенного АД и атеросклеротического поражения сосудов. Тем не менее остается неясным, имеют ли определенные цитокины (или группы цитокинов) независимое прогностическое значение в отношении долгосрочного риска развития сердечно-сосудистых событий у больных ЭАГ ॥ стадии. Целью исследования было изучить корреляцию содержания в сыворотке периферической крови цитокинов у больных ЭАГ ІІ стадии с частотой развития осложнений (ИМ, ОНМК, ТНМК) в последующие 5 лет наблюдения.

\section{ПАЦИЕНТЫ И МЕТОДЫ}

Исследование проводили на базе Регионального сосудистого центра ГБУЗ РМ «Республиканская клиническая больница № 4», ГБУЗ РМ «Республиканская клиническая больница № 3» и кафедре иммунологии, микробиологии, вирусологии ФГБОУ ВО «НИ МГУ им. Н.П. Огарева» в период 20132018 гг. Обследовали 490 больных ЭАГ ॥ стадии, из них 200 пациентов с сопоставимой гипотензивной терапией наблюдали в динамике в течение 5 лет (100 женщин и 100 мужчин, средний возраст пациентов на момент начала исследования - 57,5 \pm 1,2 года).

Критерии включения пациентов в исследование: ЭАГ II стадии; длительность заболевания 10-14 лет; трудоспособный возраст (женщины до 60 лет, мужчины до 65 лет); сопоставимые схемы базовой терапии после начала исследования (монотерапия ИАПФ или в комбинации с диуретиками); подписание пациентом добровольного информированного согласия. Критерии исключения из исследования: ассоциированные клинические состояния; сахарный диабет I типа; метаболический синдром; аутоиммунные заболевания, аллергические заболевания; симптоматическая артериальная гипертензия; перенесенные за месяц до начала исследования инфекционные и психические заболевания; алкогольная/наркотическая зависимость; отказ пациента от долгосрочного участия в исследовании. С целью определения прогнозируемого потенциала цитокинов в отношении развития ИМ, ОНМК в последующие 5 лет у 200 пациентов с ЭАГ ॥ стадии (100 мужчин и 100 женщин) в сыворотке периферической крови на момент начала исследования определяли

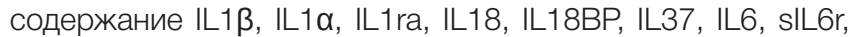
LIF, sLIFr, IGF-1, IGFBP-1, TNF $\alpha$, sTNF-RI, sVCAM-1, IL17, IL2, IL4, IL10, TGF- 1 1, IL8, CX3CL1, CXCL10, INFy, M-CSF, IL34, VEGF-A, эритропоэтина, а также вазоактивных пептидов: NO, iNOS, eNOS, ADMA, SDMA, Nt-proCNP, NtproBNP. Выбор цитокинов обоснован лиганд-рецепторным принципом реализации их эффектов - вышеуказанные цитокины синтезируются клетками сосудистого комплекса и/или органы-мишени обладают рецепторами к ним. Уровень цитокинов сыворотки периферической крови и вазоактивных веществ определяли иммуноферментным методом. В течение последующих 5 лет участников исследования опрашивали по телефону в соответствии с разработанным опросником, фиксировали случаи развития осложнений (ИМ, ОНМК, ТНМК) с последующим сопоставлением с данными амбулаторной карты.

Для статистической обработки данных использовали пакет прикладных программ Statistica 8.0 (StatSoft; версия 8.0). Нормальность распределения показателей определяли с помощью одновыборочного критерия КолмогороваСмирнова. Полученные данные представлены в виде среднего арифметического (M), стандартного отклонения (SD) и 75\% доверительного интервала для среднего (95\% ДИ) при нормальном распределении показателей. Для сравнения основных групп использовали $t$-критерий Стьюдента. Рассчитывали абсолютный и относительный риски развития повреждения органов-мишеней (ИМ, ОНМК, ТНМК) и 95\% ДИ с определением чувствительности (Se) и специфичности (Sp). Проводили построение регрессионной модели Кокса с однофакторным и многофакторным анализом.

\section{РЕЗУЛЬТАТЫ ИССЛЕДОВАНИЯ}

Проведено сопоставление уровней IL1 $\beta$, IL1 $\alpha$, IL1ra, IL37, IL18, IL18BP, IL6, sIL6r, LIF, SLIFr, TNF $\alpha$, sTNF-RI, sVCAM, IL2, IL8, IL4, IL10, IFNy, IGF-1, IGFBP-1, M-CSF, IL34, VEGF-A, CX3CL1, CXCL10, TGFß1, IL17A, эритропоэтина В сыворотке периферической крови 200 пациентов с ЭАГ II стадии (на фоне гипотензивной терапии и длительности заболевания 10-14 лет) в зависимости от развития сердечно-сосудистых осложнений в последующие 5 лет наблюдения. Ретроспективный анализ показал предикторное снижение в сыворотке периферической крови содержания IL1ra ( $p<0,001)$ и IL10 ( $p<0,001)$, повышение концентраций IL1 $\beta$ ( $p<0,001)$, TNF $\alpha(p<0,001)$, M-CSF $(p<0,001)$ в группе пациентов с развившимися в последующие 5 лет наблюдения сердечно-сосудистыми осложнениями (47 пациентов: из них у 20 больных ИМ, 14 - ОНМК, 13 - ТНМК) при сравнении с группой без осложнений за анализируемый период (табл. 1). При этом значимых различий средних уровней остальных показателей в группах с осложнениями и без осложнений не было выявлено ( $p>0,05)$.

Дальнейший интерквартильный анализ распределения числа больных с ЭАГ с развитием осложнений через 6-10 лет наблюдения с учетом уровней в сыворотке крови IL1ra, IL10, IL1ß, IL6, TNFa, M-CSF показал (табл. 2), что при содержании: IL1ra < 513 пг/мл частота развития осложнений в течение последующих 5 лет возросла в 2,24 раза $(p<0,05)$ и составила $45 \%(\mathrm{Sp}-83 \%$, Se - 41,7\%), критерий $\chi^{2}=5,25$ ( $\left.p<0,05\right), C=0,33$ (связь средней силы); IL10 < 26,3 пг/мл - рост риска в 1,94 раза $(p<0,05), 31 \%$ $\left(\mathrm{Sp}-54,9 \%\right.$, Se - 66\%), критерий $\chi^{2}=6,26(p<0,05)$, $\mathrm{C}=0,17$ (связь слабая); IL1 $\beta>18,7$ пг/мл —риск увеличился в 2,37 раза $(p<0,05)$ и был равен 38\% (Sp - 59\%, Se - 80\%), критерий $\chi^{2}=7,59(p<0,05), C=0,22$ (связь средней силы); IL6 > 23,8 - увеличение частоты в 1,76 раза $(p<0,05)$ и, соответственно, 30\% (Sp - 54\%, Se - 63,8\%), критерий $\chi^{2}=6,45$ ( $>$ < 0,05), $\mathrm{C}=0,17$ (связь слабая). При концентрации M-CSF в сыворотке периферической крови в интервале (389-453) пг/мл на 5-м году исследования частота развития 
осложнений (ОНМК, ТНМК, ИМ) в течение последующих 5 лет возросла в 3,87 раза $(p<0,001)$ по сравнению с уровнями < 389 пг/мл и > 453 пг/мл, что составило 62\% (Sp-81,6\%, Se - 66\%), критерий $\chi^{2}=32,5$ (p < 0,001), C = 0,6 (связь относительно сильная), уровень этого показателя был наиболее выраженным фактором риска по сравнению с другими цитокинами. Интерквартильное сопоставление распределения числа больных с осложнениями на 6-10-й год наблюдения при ЭАГ с учетом уровней в сыворотке крови TNFa на 5-м году исследования достоверных связей между анализируемыми переменными не выявило.

Проведенный односракторный анализ (регрессионная модель Кокса) значимости зарегистрированных изменений содержания цитокинов в сыворотке крови больных ЭАГ ॥ стадии (IL1ß > 18,8 пг/мл; IL1ra < 511 пг/мл; IL6 > 23,8 пг/мл; IL10 < 26,3 пг/мл; 389 пг/Мл < M-CSF < 453 пг/Мл) в отношении развития сердечно-сосудистых осложнений в последующие 5 лет подтвердил достоверную связь (табл. 3).

Анализ связей всех изучавшихся показателей с вазоактивными веществами (табл. 4, 5) показал, что цитокины с потенциальной предикторной информативностью в отношении риска развития осложнений у больных с ЭАГ II стадии в последующие 5 лет (IL1ra, IL10, IL1ß, TNFa, M-CSF), по данным нашего исследования, относятся к группе коррелирующих с содержанием в сыворотке периферической крови вазоактивного вещества - асимметричного диметиларгинина (ADMA). Поэтому при построении регрессионной модели Кокса мы включили ADMA в перечень многофакторного регрессионного анализа для оценки независимости выявленных критериев риска. Это имеет патогенетическое значение и влияет на диагностическую и клиническую ценность зарегистрированных изменений. Концентрация M-CSF в сыворотке периферической крови больных ЭАГ ॥ стадии имеет наибольшую корреляцию с уровнем ADMA. Также в систему многофракторного корреляционного анализа были введены классические факторы риска в соответствии с международными и российскими рекомендациями для определения степени риска развития осложнений при гипертензии. По результатам многофакторного анализа, повышение частоты повреждения органов-мишеней при ЭАГ ॥ стадии в последующий пятилетний период в исследуемой группе было связано с уровнем M-CSF в интервале (389-453) пг/мл ( 0 0,001) вне зависимости от гендерной принадлежности, а также с классическим фактором риска - уровнем ЛПНП > 3,0 ммоль/л $(p<0,01)$ (табл. 6).

\section{ОБСУЖДЕНИЕ РЕЗУЛЬТАТОВ}

По данным международных исследований, определяются значимые связи между развитием таких осложнений ЭАГ, как

Таблица 1. Уровни цитокинов при ЭАГ II стадии в зависимости от развития осложнений на фоне гипотензивной терапии за последующие 5 лет наблюдения — М (б)

\begin{tabular}{|c|c|c|}
\hline $\begin{array}{l}\text { Уровни цитокинов } \\
\text { (пг/мл) }\end{array}$ & $\begin{array}{c}\text { Больные без осложнений } \\
\qquad n=153\end{array}$ & $\begin{array}{c}\text { Больные с ЭАГ и осложнениями } \\
\qquad n=47\end{array}$ \\
\hline IL1 $\beta$ & $14,2(4,42)$ & $21,3(3,32)^{\star \text { кез осложнений }}$ \\
\hline IL1 $\alpha$ (ж) & $12,6(3,21)$ & $13,2(2,91)$ \\
\hline IL1ra & $650(112)$ & $496(93)^{\star \text { кез осложнений }}$ \\
\hline IL18 & $360(64)$ & $393(87)$ \\
\hline IL18BP & $6790(1170)$ & $6440(1620)$ \\
\hline IL6 & $21,7(4,94)$ & $24,9(4,41)^{\wedge \text { без осложнений }}$ \\
\hline sIL6r & $1889(323)$ & $1733(312)$ \\
\hline TNFa & $20,2(4,47)$ & 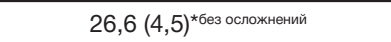 \\
\hline sTNF-RI & $2598(680)$ & $2873(699)$ \\
\hline sVCAM-1 & 577 (101) & $591(90)$ \\
\hline IL2 & $10,6(3,16)$ & $10,9(3,02)$ \\
\hline IL8 & $28,7(6,74)$ & $30,6(7,16)$ \\
\hline IL4 & $19,8(4,11)$ & $20,8(4,05)$ \\
\hline IL10 & $29,3(6,99)$ & $23,8(7,17)^{\text {*без осложнений }}$ \\
\hline IFNy & $18,4(4,18)$ & $18,1(4,39)$ \\
\hline IL37 & $93,2(26,9)$ & $90,1(24,2)$ \\
\hline IL17A & $2,5(0,56)$ & $2,46(0,49)$ \\
\hline LIF (женщины) & $7,28(2,78)$ & $7,76(2,63)$ \\
\hline sLIFr (женщины) & $40500(1120)$ & $42100(1600)$ \\
\hline IGF-1 & $116000(32300)$ & $122000(30800)$ \\
\hline M-CSF & $352(88)$ & $456(69)^{\star 6 е з ~ о с л о ж н е н и и ̆ ~}$ \\
\hline IL34 & $133(40)$ & $137(36)$ \\
\hline VEGF-A & $339(101)$ & $344(95)$ \\
\hline CX3CL1 & $510(105)$ & $542(120)$ \\
\hline CXCL10 & $17,8(4,33)$ & $18,9(3,92)$ \\
\hline TGF $\beta 1$ & $21,8(4,57)$ & $22,1(4,24)$ \\
\hline Неоптерин & $8,81(3,19)$ & $8,23(2,8)$ \\
\hline Эритропоэтин & $11,4(3,64)$ & 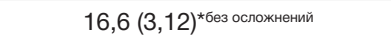 \\
\hline
\end{tabular}

Примечание: ^ $-p<0,01 ;{ }^{*}-p<0,001$ при сравнении с группой без осложнений за 5 лет наблюдения. 
ОНМК, ИМ, и содержанием в сыворотке периферической крови ряда цитокинов: IL17, IFNy, TNFa, IL6 [2, 11], sTNF-RI [9], представителей семейства IL1 $[12,13]$, хемокинов CXCсемейства [6], LIF [14], семейства IL12 [15] и др. Однако на сегодняшний день проведено ограниченное количество длительных исследований на эту тему и проанализирована динамика малого спектра цитокинов. Учитывая малое число исследований, посвященных роли цитокинов в патогенезе ЭАГ, важно не только изучить динамику того или иного иммунорегуляторного показателя при развитии осложнений. Необходимо определить факторы, сохраняющие статистическую и патогенетическую значимость при введении в патогенетическую модель других цитокинов и классических факторов, рекомендованных

Таблица 2. Отношение изменения уровней IL 1ra, IL1ß, IL6, TNFa, M-CSF, IL10 (пг/мл) в сыворотке периферической крови (начало исследования) и частоты развития осложнений (95\% ДИ) у больных с ЭАГ (длительность 10-14 лет, гипотензивная терапия) в течение последующих 5 лет

\begin{tabular}{|c|c|c|c|c|}
\hline & I квартиль & II квартиль & III квартиль & IV квартиль \\
\hline & $n=50$ & $n=50$ & $n=50$ & $n=50$ \\
\hline IL1ra & $(330-511)$ & $(512-575)$ & $(574-633)$ & $(634-820)$ \\
\hline Осложнения (человек) & 18 & 9 & 10 & 10 \\
\hline \multirow[t]{2}{*}{ Абсолютный риск (\%) } & $\begin{array}{c}36 \\
{[22,7-49,3]}\end{array}$ & $\begin{array}{c}18 \\
{[7,35-28,6]}\end{array}$ & $\begin{array}{c}20 \\
{[8,91-31]}\end{array}$ & $\begin{array}{c}20 \\
{[8,91-31]}\end{array}$ \\
\hline & $36[22,7-49,3]$ & \multicolumn{3}{|c|}{$19,3[13-25,6]$} \\
\hline Отношение рисков & \multicolumn{4}{|c|}{ I / II + III + IV квартили: 1,86 [1,13-3,05] } \\
\hline IL1 $\beta$ & $(2,95-14,8)$ & $(14,9-8,7)$ & $(18,8-22,5)$ & $(22,6-34,4)$ \\
\hline Осложнения (человек) & 1 & 8 & 18 & 20 \\
\hline \multirow{2}{*}{ Абсолютный риск (\%) } & \multirow{2}{*}{$\stackrel{2}{2}[2,43-4,43]$} & \multirow{2}{*}{$\begin{array}{c}16 \\
{[5,84-26,2]}\end{array}$} & $\begin{array}{c}36 \\
{[22,7-49,3]}\end{array}$ & $\begin{array}{c}40 \\
{[26,4-53,6]}\end{array}$ \\
\hline & & & \multicolumn{2}{|c|}{$38[28,5-47,5]$} \\
\hline Отношение рисков & \multicolumn{4}{|c|}{ II / III + IV квартили: 2,37 [1,2-4,7] $]^{*}$} \\
\hline Отношение рисков & \multicolumn{4}{|c|}{ I / II + III + IV квартили: 1,86 [1,13-3,05] } \\
\hline IL6 & $(12,5-20,8)$ & $(20,9-23,7)$ & $(23,8-27,5)$ & $(27,6-36,4)$ \\
\hline Осложнения (человек) & 8 & 9 & 14 & 16 \\
\hline \multirow{2}{*}{ Абсолютный риск (\%) } & $16[5,8-26]$ & $18[7,35-28,6]$ & $28[15,6-40]$ & 32 [19-45] \\
\hline & \multicolumn{2}{|c|}{$17[8-22,1]$} & \multicolumn{2}{|c|}{30 [21-39] } \\
\hline Отношение рисков & \multicolumn{4}{|c|}{ I + II / III + IV квартили: 1,76 [1,04-2,99] $]^{*}$} \\
\hline TNFa & $(10,3-18,3)$ & $(18,6-23,7)$ & $(21,4-24)$ & $(24,1-32,4)$ \\
\hline Осложнения (человек) & 10 & 13 & 12 & 12 \\
\hline Абсолютный риск (\%) & $20[8,9-31]$ & $26[13,8-38]$ & $24[12,2-35,8]$ & $24[12,2-35,8]$ \\
\hline Отношение рисков & \multicolumn{2}{|c|}{ I / II квартили: 1,3 [0,63-2,69] } & \multicolumn{2}{|c|}{ II / III + IV квартили: 0,92 [1,04-1,65] } \\
\hline M-CSF & $(138-319)$ & $(320-388)$ & $(389-453)$ & $(454-640)$ \\
\hline $\begin{array}{l}\text { Пациенты с осложнениями } \\
\text { (человек) }\end{array}$ & 0 & 7 & 31 & 9 \\
\hline Абсолютный риск (\%) & 0 & $14[4,42-23,6]$ & $62[48,5-55,4]$ & $18[7,55-28,6]$ \\
\hline Отношение рисков & \multicolumn{4}{|c|}{$\begin{array}{c}\text { II + IV / III квартили: 3,87 [2,35-6,38] * } \\
\text { II/IV квартили = 1,28 [0,52-3,18] }\end{array}$} \\
\hline IL10 & $(5,1-19,9)$ & $(20-26,2)$ & $(26,3-31,5)$ & $(31,6-47,5)$ \\
\hline $\begin{array}{l}\text { Пациенты с осложнениями } \\
\text { (человек) }\end{array}$ & 15 & 16 & 8 & 8 \\
\hline \multirow{2}{*}{ Абсолютный риск (\%) } & 30 [19-45] & $32[15,6-40]$ & $16[5,84-26,2]$ & $16[5,84-26,2]$ \\
\hline & \multicolumn{2}{|c|}{31 [22-40] } & \multicolumn{2}{|c|}{$16[5,84-26,2]$} \\
\hline Отношение рисков & & $I+I I / I I I+I V$ & {$[1,13-3,31]^{\star}$} & \\
\hline
\end{tabular}

Примечание: * - $p<0,05$ при сравнении абсолютных рисков, если интервал не включает 1.

Таблица 3. Анализ влияния содержания IL1ß, IL 1ra, IL6, IL10, M-CSF на частоту развития осложнений (95\% ДИ) в течение последующих 5 лет наблюдения у больных с ЭАГ ॥ стадии и регрессионная модель Кокса, однофакторный анализ

\begin{tabular}{|l|c|c|c|c|c|}
\hline \multicolumn{1}{|c|}{ Переменные } & Beta & Standard & $t$-value & Exponent Beta & $p$ \\
\hline IL13 (> 18,8 пг/мл) & 1,99 & 0,35 & 5,68 & 2,37 & 0,006 \\
\hline IL1ra (<511 пг/мл) & 1,24 & 0,28 & 4,43 & 2,06 & 0,009 \\
\hline IL6 (> 23,8 пг/мл) & 1,27 & 0,35 & 3,62 & 1,89 & 0,042 \\
\hline IL10 (<26,3 пг/мл) & 1,22 & 0,27 & 4,52 & 1,91 & 0,007 \\
\hline M-CSF (389-453) пг/мл & 2,44 & 0,25 & 9,76 & 3,89 & 0,0004 \\
\hline
\end{tabular}

Примечание: данные модели Кокса: Beta - коэффициент регрессии; Standard - стандартная ошибка коэффициента регрессии; $t$-value - значение t-критерия для оценки коэффициента регрессии; Exponent Beta - значения относительного риска, связь с интервалом изменения анализируемого фактора; $p$ - уровень значимости. 
для расчета риска развития осложнений у данной категории больных. Полученные в ходе исследования данные показали, что изменения содержания в сыворотке периферической крови больных исследуемой группы IL1ra, IL10, IL1ß, IL6, M-CSF были связаны с развитием осложнений (ИМ, ОНМК, ТНМК) в последующие 5 лет. Повышение уровней противовоспалительных цитокинов IL1ra, IL10 коррелировало с меньшим числом осложнений Корреляции уровней остальных анализируемых цитокинов с риском развития осложнений ЭАГ не выявлено. Есть расхождения с некоторыми данными литературы, указывающими на связь LIF, IL $1 \alpha$ и др. с частотой осложнений $[10,14]$. Это объясняется различиями критериев включения в разные исследования (гендерное соотношение, возраст, сопоставимая гипотензивная терапия, достижение целевых уровней АД и др.) и подчеркивает высокую значимость степени однородности группы наблюдения, влияющую на патогенетическую значимость полученных данных. Важно отметить, что, по результатам нашего исследования, только цитокины, коррелирующие с содержанием ADMA, являются потенциальными предикторами повреждения органовмишеней у больных ЭАГ II стадии при длительности патологии 10-14 лет (на фоне гипотензивной терапии). ADMA метилированный аналог L-аргинина (субстрата для синтеза NO), конкурентно ингибирует функциональную активность eNOS [16], что снижает образование NO и его доступность для вазорелаксации и вазопротекции [17]. Данная патогенетическая цепочка важна, на сегодняшний день многие научные платсормы подчеркивают актуальность расширения информации о роли ADMA и SDMA в развитии патологических процессов. В ходе работы определено, что M-CSF является единственным независимым критерием из спектра анализируемых цитокинов, обладающим высокой предикторной информативностью в отношении повышения риска развития ОНМК, ИМ, ТНМК у больных ЭАГ ॥ стадии при длительности патологии 10-14 лет (на

Таблица 4. Корреляционная матрица цитокинов сыворотки периферической крови и вазоактивных веществ при ЭАГ II стадии (длительность 10-14 лет) на фоне гипотензивной терапии

\begin{tabular}{|c|c|c|c|c|c|c|c|c|c|c|c|c|c|}
\hline & IL37 & LIF & sLIFr & IGF-1 & IGFBP-1 & M-CSF & IL34 & VEGF-A & CX3CL1 & CXCL10 & TGF $\beta 1$ & IL17A & Эритропоэтин \\
\hline AT II & $\begin{array}{c}-0,28 \\
p>0,05\end{array}$ & $\begin{array}{c}0,51 \\
p<0,05\end{array}$ & $\begin{array}{c}-0,36 \\
p>0,05\end{array}$ & $\begin{array}{c}0,23 \\
p>0,05\end{array}$ & $\begin{array}{c}0,23 \\
p>0,05\end{array}$ & $\begin{array}{c}0,34 \\
p>0,05\end{array}$ & $\begin{array}{c}0,24 \\
p>0,05\end{array}$ & $\begin{array}{c}0,29 \\
p>0,05\end{array}$ & $\begin{array}{c}0,12 \\
p>0,05\end{array}$ & $\begin{array}{c}0,31 \\
p>0,05\end{array}$ & $\begin{array}{c}0,23 \\
p>0,05\end{array}$ & $\begin{array}{c}0,21 \\
p>0,05\end{array}$ & $\begin{array}{c}0,23 \\
p>0,05\end{array}$ \\
\hline ET-1 & $\begin{array}{c}-0,41 \\
p>0,05\end{array}$ & $\begin{array}{c}0,31 \\
p>0,05\end{array}$ & $\begin{array}{c}0,28 \\
p>0,05\end{array}$ & $\begin{array}{c}0,21 \\
p>0,05\end{array}$ & $\begin{array}{c}0,22 \\
p>0,05\end{array}$ & $\begin{array}{c}0,43 \\
p>0,05\end{array}$ & $\begin{array}{c}0,21 \\
p>0,05\end{array}$ & $\begin{array}{c}0,42 \\
p>0,05\end{array}$ & $\begin{array}{c}0,27 \\
p>0,05\end{array}$ & $\begin{array}{c}0,35 \\
p>0,05\end{array}$ & $\begin{array}{c}0,31 \\
p>0,05\end{array}$ & $\begin{array}{c}0,23 \\
p>0,05\end{array}$ & $\begin{array}{c}0,32 \\
p>0,05\end{array}$ \\
\hline NO & $\begin{array}{c}0,69 \\
p<0,05\end{array}$ & $\begin{array}{c}0,42 \\
p>0,05\end{array}$ & $\begin{array}{c}0,41 \\
p>0,05\end{array}$ & $\begin{array}{c}0,23 \\
p>0,05\end{array}$ & $\begin{array}{c}0,18 \\
p>0,05\end{array}$ & $\begin{array}{c}0,32 \\
p>0,05\end{array}$ & $\begin{array}{c}0,31 \\
p>0,05\end{array}$ & $\begin{array}{c}0,34 \\
p>0,05\end{array}$ & $\begin{array}{c}0,31 \\
p>0,05\end{array}$ & $\begin{array}{c}0,39 \\
p>0,05\end{array}$ & $\begin{array}{c}0,48 \\
p>0,05\end{array}$ & $\begin{array}{c}0,21 \\
p>0,05\end{array}$ & $\begin{array}{c}0,23 \\
p>0,05\end{array}$ \\
\hline ADMA & $\begin{array}{c}-0,32 \\
p>0,05\end{array}$ & $\begin{array}{c}0,33 \\
p>0,05\end{array}$ & $\begin{array}{c}0,49 \\
p>0,05\end{array}$ & $\begin{array}{c}0,28 \\
p>0,05\end{array}$ & $\begin{array}{c}0,45 \\
p>0,05\end{array}$ & $\begin{array}{c}0,58 \\
p<0,05\end{array}$ & $\begin{array}{c}0,24 \\
p>0,05\end{array}$ & $\begin{array}{c}0,28 \\
p>0,05\end{array}$ & $\begin{array}{c}0,22 \\
p>0,05\end{array}$ & $\begin{array}{c}0,15 \\
p>0,05\end{array}$ & $\begin{array}{c}0,34 \\
p>0,05\end{array}$ & $\begin{array}{c}0,31 \\
p>0,05\end{array}$ & $\begin{array}{c}0,32 \\
p>0,05\end{array}$ \\
\hline SDMA & $\begin{array}{c}-0,78 \\
p<0,001\end{array}$ & $\begin{array}{c}0,28 \\
p>0,05\end{array}$ & $\begin{array}{c}0,45 \\
p>0,05\end{array}$ & $\begin{array}{c}0,38 \\
p>0,05\end{array}$ & $\begin{array}{c}0,27 \\
p>0,05\end{array}$ & $\begin{array}{c}0,52 \\
p<0,05\end{array}$ & $\begin{array}{c}0,41 \\
p>0,05\end{array}$ & $\begin{array}{c}0,31 \\
p>0,05\end{array}$ & $\begin{array}{c}0,52 \\
p<0,05\end{array}$ & $\begin{array}{c}0,5 \\
p<0,05\end{array}$ & $\begin{array}{c}0,26 \\
p>0,05\end{array}$ & $\begin{array}{c}0,31 \\
p>0,05\end{array}$ & $\begin{array}{c}0,21 \\
p>0,05\end{array}$ \\
\hline eNOS & $\begin{array}{c}0,32 \\
p>0,05\end{array}$ & $\begin{array}{c}0,38 \\
p>0,05\end{array}$ & $\begin{array}{c}-0,39 \\
p>0,05\end{array}$ & $\begin{array}{c}0,21 \\
p>0,05\end{array}$ & $\begin{array}{c}0,34 \\
p>0,05\end{array}$ & $\begin{array}{c}0,31 \\
p>0,05\end{array}$ & $\begin{array}{c}0,24 \\
p>0,05\end{array}$ & $\begin{array}{c}0,11 \\
p>0,05\end{array}$ & $\begin{array}{c}-0,18 \\
p>0,05\end{array}$ & $\begin{array}{c}-0,31 \\
p>0,05\end{array}$ & $\begin{array}{c}0,23 \\
p>0,05\end{array}$ & $\begin{array}{c}0,32 \\
p>0,05\end{array}$ & $\begin{array}{c}0,34 \\
p>0,05\end{array}$ \\
\hline iNOS & $\begin{array}{c}-0,41 \\
p>0,05\end{array}$ & $\begin{array}{c}0,28 \\
p>0,05\end{array}$ & $\begin{array}{c}0,45 \\
p>0,05\end{array}$ & $\begin{array}{c}0,34 \\
p>0,05\end{array}$ & $\begin{array}{c}0,38 \\
p>0,05\end{array}$ & $\begin{array}{c}0,17 \\
p>0,05\end{array}$ & $\begin{array}{c}0,21 \\
p>0,05\end{array}$ & $\begin{array}{c}0,21 \\
p>0,05\end{array}$ & $\begin{array}{c}0,69 \\
p<0,01\end{array}$ & $\begin{array}{c}0,71 \\
p<0,01\end{array}$ & $\begin{array}{c}0,34 \\
p>0,05\end{array}$ & $\begin{array}{c}0,21 \\
p>0,05\end{array}$ & $\begin{array}{c}0,21 \\
p>0,05\end{array}$ \\
\hline $\begin{array}{l}\text { NT- } \\
\text { proCNP }\end{array}$ & $\begin{array}{c}-0,27 \\
p>0,05\end{array}$ & $\begin{array}{c}0,41 \\
p>0,05\end{array}$ & $\begin{array}{c}-0,28 \\
p>0,05\end{array}$ & $\begin{array}{c}0,27 \\
p>0,05\end{array}$ & $\begin{array}{c}0,37 \\
p>0,05\end{array}$ & $\begin{array}{c}0,41 \\
p>0,05\end{array}$ & $\begin{array}{c}0,25 \\
p>0,05\end{array}$ & $\begin{array}{c}0,32 \\
p>0,05\end{array}$ & $\begin{array}{c}-0,31 \\
p>0,05\end{array}$ & $\begin{array}{c}-0,27 \\
p>0,05\end{array}$ & $\begin{array}{c}0,52 \\
p<0,05\end{array}$ & $\begin{array}{c}0,23 \\
p>0,05\end{array}$ & $\begin{array}{c}0,24 \\
p>0,05\end{array}$ \\
\hline $\begin{array}{c}\text { NT- } \\
\text { proBNP }\end{array}$ & $\begin{array}{c}-0,78 \\
p<0,01\end{array}$ & $\begin{array}{c}-0,65 \\
p<0,05\end{array}$ & $\begin{array}{c}0,31 \\
p>0,05\end{array}$ & $\begin{array}{c}0,31 \\
p>0,05\end{array}$ & $\begin{array}{c}0,24 \\
p>0,05\end{array}$ & $\begin{array}{c}0,45 \\
p>0,05\end{array}$ & $\begin{array}{c}0,18 \\
p>0,05\end{array}$ & $\begin{array}{c}0,34 \\
p>0,05\end{array}$ & $\begin{array}{c}0,21 \\
p>0,05\end{array}$ & $\begin{array}{c}0,2 \\
p>0,05\end{array}$ & $\begin{array}{c}0,33 \\
p>0,05\end{array}$ & $\begin{array}{c}0,27 \\
p>0,05\end{array}$ & $\begin{array}{c}0,12 \\
p>0,05\end{array}$ \\
\hline
\end{tabular}

Примечание: данные представлены в виде коэффицинта множественной корреляции, знак указывает направление связи, p - уровень значимости.

Таблица 5. Корреляционная матрица цитокинов сыворотки периферической крови и вазоактивных веществ при ЭАГ II стадии (длительность 10-14 лет) на фоне гипотензивной терапии

\begin{tabular}{|c|c|c|c|c|c|c|c|c|c|c|c|c|c|c|c|}
\hline & IL1 $\beta$ & IL1 $\alpha$ & IL1ra & IL18 & IL18BP & IL6 & slL6r & TNFa & sTNF-RI & sVCAM-1 & IL2 & IL8 & IL4 & IL10 & IFNy \\
\hline AT II & $\begin{array}{c}0,41 \\
p>0,05\end{array}$ & $\begin{array}{c}0,34 \\
p>0,05\end{array}$ & $\begin{array}{c}-0,19 \\
p>0,05\end{array}$ & $\begin{array}{c}0,41 \\
p>0,05\end{array}$ & $\begin{array}{c}-0,23 \\
p>0,05\end{array}$ & $\begin{array}{c}0,43 \\
p>0,05\end{array}$ & $\begin{array}{c}0,33 \\
p>0,05\end{array}$ & $\begin{array}{c}0,41 \\
p>0,05\end{array}$ & $\begin{array}{c}0,22 \\
p>0,05\end{array}$ & $\begin{array}{c}0,21 \\
p>0,05\end{array}$ & $\begin{array}{c}0,18 \\
p>0,05\end{array}$ & $\begin{array}{c}0,37 \\
p>0,05\end{array}$ & $\begin{array}{c}0,22 \\
p>0,05\end{array}$ & $\begin{array}{c}-0,62 \\
p<0,05\end{array}$ & $\begin{array}{c}0,16 \\
p>0,05\end{array}$ \\
\hline ET-1 & $\begin{array}{c}0,68 \\
p<0,05\end{array}$ & $\begin{array}{c}0,65 \\
p<0,05\end{array}$ & $\begin{array}{c}-0,62 \\
p<0,05\end{array}$ & $\begin{array}{c}0,34 \\
p>0,05\end{array}$ & $\begin{array}{c}-0,36 \\
p>0,05\end{array}$ & $\begin{array}{c}0,27 \\
p>0,05\end{array}$ & $\begin{array}{c}0,41 \\
p>0,05\end{array}$ & $\begin{array}{c}0,36 \\
p>0,05\end{array}$ & $\begin{array}{c}0,24 \\
p>0,05\end{array}$ & $\begin{array}{c}0,31 \\
p>0,05\end{array}$ & $\begin{array}{c}0,25 \\
p>0,05\end{array}$ & $\begin{array}{c}0,69 \\
p<0,01\end{array}$ & $\begin{array}{c}0,24 \\
p>0,05\end{array}$ & $\begin{array}{c}-0,36 \\
p>0,05\end{array}$ & $\begin{array}{c}0,18 \\
p>0,05\end{array}$ \\
\hline NO & $\begin{array}{c}0,64 \\
p<0,05\end{array}$ & $\begin{array}{c}0,46 \\
p>0,05\end{array}$ & $\begin{array}{c}0,49 \\
p<0,05\end{array}$ & $\begin{array}{c}-0,27 \\
p>0,05\end{array}$ & $\begin{array}{c}0,64 \\
p<0,05\end{array}$ & $\begin{array}{c}0,49 \\
p>0,05\end{array}$ & $\begin{array}{c}0,33 \\
p>0,05\end{array}$ & $\begin{array}{c}0,44 \\
p>0,05\end{array}$ & $\begin{array}{c}-0,38 \\
p>0,05\end{array}$ & $\begin{array}{c}0,25 \\
p>0,05\end{array}$ & $\begin{array}{c}0,37 \\
p>0,05\end{array}$ & $\begin{array}{c}-0,41 \\
p>0,05\end{array}$ & $\begin{array}{c}-0,38 \\
p>0,05\end{array}$ & $\begin{array}{c}0,33 \\
p>0,05\end{array}$ & $\begin{array}{c}0,41 \\
p>0,05\end{array}$ \\
\hline ADMA & $\begin{array}{c}0,52 \\
p<0,05\end{array}$ & $\begin{array}{c}0,4 \\
p>0,05\end{array}$ & $\begin{array}{c}-0,58 \\
p<0,05\end{array}$ & $\begin{array}{c}0,36 \\
p>0,05\end{array}$ & $\begin{array}{c}-0,41 \\
p>0,05\end{array}$ & $\begin{array}{c}0,57 \\
p<0,05\end{array}$ & $\begin{array}{c}0,25 \\
p>0,05\end{array}$ & $\begin{array}{c}0,38 \\
p>0,05\end{array}$ & $\begin{array}{c}0,31 \\
p>0,05\end{array}$ & $\begin{array}{c}0,38 \\
p>0,05\end{array}$ & $\begin{array}{c}0,33 \\
p>0,05\end{array}$ & $\begin{array}{c}0,27 \\
p>0,05\end{array}$ & $\begin{array}{c}0,31 \\
p>0,05\end{array}$ & $\begin{array}{c}-0,55 \\
p<0,05\end{array}$ & $\begin{array}{c}0,24 \\
p>0,05\end{array}$ \\
\hline SDMA & $\begin{array}{c}0,34 \\
p>0,05\end{array}$ & $\begin{array}{c}0,29 \\
p>0,05\end{array}$ & $\begin{array}{c}-0,16 \\
p>0,05\end{array}$ & $\begin{array}{c}0,48 \\
p>0,05\end{array}$ & $\begin{array}{c}-0,71 \\
p<0,01\end{array}$ & $\begin{array}{c}0,29 \\
p>0,05\end{array}$ & $\begin{array}{c}0,23 \\
p>0,05\end{array}$ & $\begin{array}{c}0,26 \\
p>0,05\end{array}$ & $\begin{array}{c}0,25 \\
p>0,05\end{array}$ & $\begin{array}{c}0,43 \\
p>0,05\end{array}$ & $\begin{array}{c}0,72 \\
p<0,01\end{array}$ & $\begin{array}{c}0,53 \\
p<0,05\end{array}$ & $\begin{array}{c}0,25 \\
p>0,05\end{array}$ & $\begin{array}{c}-0,23 \\
p>0,05\end{array}$ & $\begin{array}{c}0,58 \\
p<0,05\end{array}$ \\
\hline eNOS & $\begin{array}{c}-0,62 \\
p<0,05\end{array}$ & $\begin{array}{c}-0,67 \\
p<0,05\end{array}$ & $\begin{array}{c}0,51 \\
p<0,05\end{array}$ & $\begin{array}{c}0,22 \\
p>0,05\end{array}$ & $\begin{array}{c}0,37 \\
p>0,05\end{array}$ & $\begin{array}{c}-0,22 \\
p>0,05\end{array}$ & $\begin{array}{c}-0,35 \\
p>0,05\end{array}$ & $\begin{array}{c}0,4 \\
p>0,05\end{array}$ & $\begin{array}{c}0,27 \\
p>0,05\end{array}$ & $\begin{array}{c}0,31 \\
p>0,05\end{array}$ & $\begin{array}{c}-0,13 \\
p>0,05\end{array}$ & $\begin{array}{c}-0,31 \\
p>0,05\end{array}$ & $\begin{array}{c}0,27 \\
p>0,05\end{array}$ & $\begin{array}{c}0,46 \\
p>0,05\end{array}$ & $\begin{array}{c}-0,19 \\
p>0,05\end{array}$ \\
\hline iNOS & $\begin{array}{c}0,78 \\
p<0,01\end{array}$ & $\begin{array}{c}0,49 \\
p>0,05\end{array}$ & $\begin{array}{c}-0,12 \\
p>0,05\end{array}$ & $\begin{array}{c}0,36 \\
p>0,05\end{array}$ & $\begin{array}{c}-0,39 \\
p>0,05\end{array}$ & $\begin{array}{c}0,68 \\
p<0,05\end{array}$ & $\begin{array}{c}0,56 \\
p<0,05\end{array}$ & $\begin{array}{c}0,42 \\
p>0,05\end{array}$ & $\begin{array}{c}0,41 \\
p>0,05\end{array}$ & $\begin{array}{c}0,41 \\
p>0,05\end{array}$ & $\begin{array}{c}0,62 \\
p<0,05\end{array}$ & $\begin{array}{c}0,42 \\
p>0,05\end{array}$ & $\begin{array}{c}0,41 \\
p>0,05\end{array}$ & $\begin{array}{c}-0,4 \\
p>0,05\end{array}$ & $\begin{array}{c}0,52 \\
p<0,05\end{array}$ \\
\hline $\begin{array}{l}\text { NT- } \\
\text { proCNP }\end{array}$ & $\begin{array}{c}-0,58 \\
p<0,05\end{array}$ & $\begin{array}{c}0,68 \\
p<0,05\end{array}$ & $\begin{array}{c}0,13 \\
p>0,05\end{array}$ & $\begin{array}{c}0,31 \\
p>0,05\end{array}$ & $\begin{array}{c}-0,37 \\
p>0,05\end{array}$ & $\begin{array}{c}-0,61 \\
p<0,05\end{array}$ & $\begin{array}{c}0,24 \\
p>0,05\end{array}$ & $\begin{array}{c}0,52 \\
p<0,05\end{array}$ & $\begin{array}{c}0,39 \\
p>0,05\end{array}$ & $\begin{array}{c}0,24 \\
p>0,05\end{array}$ & $\begin{array}{c}-0,74 \\
p<0,01\end{array}$ & $\begin{array}{c}-0,37 \\
p>0,05\end{array}$ & $\begin{array}{c}0,39 \\
p>0,05\end{array}$ & $\begin{array}{c}0,29 \\
p>0,05\end{array}$ & $\begin{array}{c}-0,64 \\
p<0,05\end{array}$ \\
\hline $\begin{array}{c}\text { NT } \\
\text {-proBNP }\end{array}$ & $\begin{array}{c}0,47 \\
p>0,05\end{array}$ & $\begin{array}{c}0,29 \\
p>0,05\end{array}$ & $\begin{array}{c}-0,31 \\
p>0,05\end{array}$ & $\begin{array}{c}0,14 \\
p>0,05\end{array}$ & $\begin{array}{c}-0,73 \\
p<0,01\end{array}$ & $\begin{array}{c}0,17 \\
p>0,05\end{array}$ & $\begin{array}{c}-0,12 \\
p>0,05\end{array}$ & $\begin{array}{c}0,51 \\
p<0,05\end{array}$ & $\begin{array}{c}0,24 \\
p>0,05\end{array}$ & $\begin{array}{c}0,32 \\
p>0,05\end{array}$ & $\begin{array}{c}0,32 \\
p>0,05\end{array}$ & $\begin{array}{c}0,21 \\
p>0,05\end{array}$ & $\begin{array}{c}0,24 \\
p>0,05\end{array}$ & $\begin{array}{c}-0,22 \\
p>0,05\end{array}$ & $\begin{array}{c}0,32 \\
p>0,05\end{array}$ \\
\hline
\end{tabular}

Примечание: данные представлены в виде коэффициента множественной корреляции, знак указывает направление связи; $p$ - уровень значимости. 
Таблица 6. Анализ влияния содержания IL1ß, IL1ra, IL6, IL10, M-CSF, ADMA и классических факторов риска на частоту развития осложнений (95\% ДИ) в течение последующих 5 лет наблюдения у больных с ЭАГ II стадии; регрессионная модель Кокса; многофакторный анализ

\begin{tabular}{|c|c|c|c|c|c|}
\hline Переменные & Beta & Standard & $t$-value & Exponent Beta & $p$ \\
\hline $\operatorname{IL} 1 \beta$ (> 18,8 пг/мл) & 1,19 & 0,73 & 1,63 & 2,05 & 0,058 \\
\hline IL1ra (< 511 пг/мл) & 1,04 & 0,62 & 1,67 & 1,34 & 0,067 \\
\hline IL6 (> 23,8 пг/мл) & 1,07 & 0,63 & 1,69 & 2,17 & 0,062 \\
\hline IL10 (< 26,3 пг/мл) & 1,06 & 0,66 & 1,61 & 1,32 & 0,072 \\
\hline M-CSF (389-453) пг/мл & 2,17 & 0,34 & 6,38 & 2,53 & 0,0007 \\
\hline ADMA (> 0,86 мкмоль/л) & 1,49 & 0,77 & 1,93 & 2,09 & 0,068 \\
\hline Общий холестерин > 4,9 ммоль/л & 1,18 & 0,73 & 1,62 & 1,63 & 0,062 \\
\hline ЛПНП > 3,0 ммоль /л & 1,88 & 0,43 & 4,37 & 2,28 & 0,004 \\
\hline ЛПВП у мужчин < 1,0 ммоль/л, у женщин < 1,2 ммоль/л & $1,12 / 1,19$ & $0,71 / 0,68$ & 1,58 & $1,38 / 1,32$ & $0,071 / 0,069$ \\
\hline
\end{tabular}

Примечание: данные модели Кокса; Beta - коэффициент регрессии; Standard - стандартная ошибка коэффициента регрессии; $t$-value - значение t-критерия для оценки коэффициента регрессии; Ехponent Beta - значения относительного риска, связь с интервалом изменения анализируемого фактора; $p$ - уровень значимости.

фоне гипотензивной терапии), в том числе при сравнении с ADMA и классическими факторами риска. Это еще раз подтверждает приоритет цитокина в корреляционной патогенетической модели M-CSF-ADMA с дальнейшим каскадом реакций прогрессирования. Ранее нами были опубликованы данные о прямой корреляции уровня M-CSF > 453 пг/мл и содержания в сыворотке периферической крови VEGF-A, что соотносилось по данным коронарографии с выраженным коллатеральным кровообращением в миокарде и может объяснять снижение частоты ИМ у пациентов с данными характеристиками [18], суммарно влияя на общий риск осложнений. M-CSF через рецептор M-CSFR-1 способен активировать MAP-киназы, которые играют ключевую роль в производстве VEGF-A посредством активации ERK, повышая промоторную активность р38 и JNK, стабилизируя мPHK VEGF-A, при этом данные эффекты дозозависимы [19].

\section{ВЫВОДЫ}

В ходе исследования получены данные, доказывающие, что изменения содержания цитокинов в сыворотке периферической крови больных ЭАГ ॥ стадии: IL1 >
18,8 пг/мл, IL1ra < 511 пг/мл, IL6 > 23,8 пг/мл, IL10 < 26,3 пг/Мл, коррелируют с пятилетней частотой развития осложнений (ИМ, ОНМК, ТНМК) у больных ЭАГ ІІ стадии при длительности патологии 10-14 лет (на фоне гипотензивной терапии). При этом только M-CSF в интервале 389-453 пг/мл имеет статистически независимые характеристики в отношении развития кардиальных и цереброваскулярных осложнений. Несмотря на теоретическую значимость полученных результатов, необходимо отметить, что при высокой специфичности (81\%) содержания М-CSF в интервале 389-453 пг/мл в сыворотке периферической крови больных ЭАГ ІІ стадии при длительности патологии 10-14 лет (на фоне гипотензивной терапии) в отношении формирования ИМ, ОНМК, ТНМК зарегистрирована относительно низкая чувствительность этого показателя (66\%) в рамках предикции осложнений, что требует введения дополнительного критерия, связанного с данным цитокином и повышающего диагностическую (практическую) ценность. Требуется дополнительное изучение роли индивидуальных характеристик, в частности генетического компонента (полиморфных вариантов генотипа CSF1R TC/CA rs386693509: TC/CA) в реализации выявленных связей при прогредиентном течении заболевания.

\section{Литература}

1. Wenzel U, Turner JE, Krebs C, et al. Immune Mechanisms in Arterial Hypertension. J Am Soc Nephrol. 2016; 27 (3): 677-86.

2. McMaster WG, Kirabo A, Madhur MS, Harrison DG. Inflammation, immunity, and hypertensive end organ damage. Circ Res. 2015; 116 (6): 1022-33.

3. Bennardo M, Alibhai F, Tsimakouridze E, et al. Day-night dependence of gene expression and inflammatory responses in the remodeling murine heart post-myocardial. Am J Physiol Regul Integr Comp Physiol. 2016; 311 (6): 1243-54.

4. Ji Q, Cheng $\mathrm{G}, \mathrm{Ma} \mathrm{N}$, et al. Circulating Th1, Th2, and Th17 Levels in Hypertensive Patients. Dis Markers 2017; (2017): 7146290. Available from: https://www.hindawi.com/journals/ dm/2017/7146290. PubMed PMID: 28757677.

5. Nosalski R, McGinnigle E, Siedlinski M, Guzik TJ. Novel Immune Mechanisms in Hypertension and Cardiovascular Risk. Current Cardiovascular Risk Reports. 2017; 11 (4): 12. Available from: https://www.researchgate.net/publication/314274524_Novel_ Immune_Mechanisms_in_Hypertension_and_Cardiovascular_Risk.

6. Rudemiller NP, Crowley SD. The role of chemokines in hypertension and consequent target organ damage. Pharmacological research. 2017; (119): 404-11.

7. Schwanekamp JA, Lorts A, Sargent MA, et al. TGFBI functions similar to periostin but is uniquely dispensable during cardiac injury. PLoS ONE. 2017; 12 (7). Available from: https://journals. plos.org/plosone/article?id=10.1371/journal.pone.0181945.

8. Wang $\mathrm{P}, \mathrm{He} \mathrm{Q}$, Liu C, et al. Functional polymorphism rs3783553 in the 3'-untranslated region of IL-1A increased the risk of ischemic stroke: A case-control study. Medicine. 2016; 96 (46). Available from: https://www.researchgate.net/publication/320348816_4-bp_ insertiondeletion_rs3783553_polymorphism_within_the_3aposUTR_ of_IL1A_contributes_to_the_risk_of_prostate_cancer_in_a_sample_ of_Iranian_Population.

9. Carlsson $\mathrm{AC}$, Jansson $\mathrm{JH}$, Söderberg $\mathrm{S}$, et al. Levels of soluble tumor necrosis factor receptor 1 and 2, gender, and risk of myocardial infarction in Northern Sweden. Atherosclerosis. 2018; (272): 41-6.

10. Huang S, Frangogiannis NG. Anti-inflammatory therapies in myocardial infarction: failures, hopes and challenges. $\mathrm{Br} J$ Pharmacol. 2018; 175 (9): 1377-400.

11. Itani HA, Harrison DG. Memories that last in hypertension. Am J Physiol Renal Physiol. 2015; 308 (11): F1197-F1199. DOl: 10.1152/ajprenal.00633.2014. 
12. Rucker JA, Crowley SD. The role of macrophages in hypertension and its complications Pflugers Arch. 2017; 469 (3-4): 419-30.

13. Hartman MHT, Groot HE, et al. Translational overview of cytokine inhibition in acute myocardial infarction and chronic heart failure. Trends Cardiovasc Med. 2018; 28 (6). DOI: 10.1016/j. tcm.2018.02.003. Available from: https://www.researchgate.net/ publication/260757993_Heart_Failure.

14. Jia D, Cai M, XiY, et al. Interval exercise training increases LIF expression and prevents myocardial infarction-induced skeletal muscle atrophy in rats. Life Sci. 2018; (193): 77-86.

15. Van der Heijden T, Bot I, Kuiper J. The IL-12 cytokine family in cardiovascular diseases. Cytokine. 2017; S1043-4666(17)303150. DOI: 10.1016/j.cyto.2017.10.010.

16. Papageorgiou N, Androulakis E, Papaioannou S, et al Homoarginine in the shadow of asymmetric dimethylarginine: from nitric oxide to cardiovascular disease. Amino Acids. 2015; 47 (9): $1741-50$

\section{References}

1. Wenzel U, Turner JE, Krebs C, et al. Immune Mechanisms in Arterial Hypertension. J Am Soc Nephrol. 2016; 27 (3): 677-86.

2. McMaster WG, Kirabo A, Madhur MS, Harrison DG. Inflammation, immunity, and hypertensive end organ damage. Circ Res. 2015; 116 (6): 1022-33.

3. Bennardo M, Alibhai F, Tsimakouridze E, et al. Day-night dependence of gene expression and inflammatory responses in the remodeling murine heart post-myocardial. Am J Physiol Regu Integr Comp Physiol. 2016; 311 (6): 1243-54.

4. Ji Q, Cheng G, Ma N, et al. Circulating Th1, Th2, and Th17 Levels in Hypertensive Patients. Dis Markers 2017; (2017): 7146290. Available from: https://www.hindawi.com/journals/ dm/2017/7146290. PubMed PMID: 28757677.

5. Nosalski R, McGinnigle E, Siedlinski M, Guzik TJ. Novel Immune Mechanisms in Hypertension and Cardiovascular Risk. Current Cardiovascular Risk Reports. 2017; 11 (4): 12. Available from: https://www.researchgate.net/publication/314274524_Novel Immune_Mechanisms_in_Hypertension_and_Cardiovascular_Risk.

6. Rudemiller NP, Crowley SD. The role of chemokines in hypertension and consequent target organ damage. Pharmacological research. 2017; (119): 404-11.

7. Schwanekamp JA, Lorts A, Sargent MA, et al. TGFBI functions similar to periostin but is uniquely dispensable during cardiac injury. PLoS ONE. 2017; 12 (7). Available from: https://journals. plos.org/plosone/article?id=10.1371/journal. pone.0181945.

8. Wang $\mathrm{P}, \mathrm{He} \mathrm{Q}$, Liu C, et al. Functional polymorphism rs3783553 in the 3'-untranslated region of IL-1A increased the risk of ischemic stroke: A case-control study. Medicine. 2016; 96 (46). Available from: https://www.researchgate.net/publication/320348816_4-bp_ insertiondeletion_rs3783553_polymorphism_within_the_3aposUTR_ of_IL1A_contributes_to_the_risk_of_prostate_cancer_in_a_sample_ of_Iranian_Population.

9. Carlsson AC, Jansson JH, Söderberg S, et al. Levels of soluble tumor necrosis factor receptor 1 and 2, gender, and risk of myocardial infarction in Northern Sweden. Atherosclerosis. 2018; (272): 41-6.
17. Shin S, Thapa SK, Fung H-L. Cellular interactions between L-arginine and asymmetric dimethylarginine: Transport and metabolism. PLoS One. 2017; 12 (5): e0178710. DOI: 10.1371/ journal.pone.0178710.

18. Радаева О. А., Симбирцев А. С. M-CSF, IL-34, VEGF-A как факторы риска развития инфаркта миокарда, острого нарушения мозгового кровообращения у больных эссенциальной артериальной гипертензией. Российский иммунологический журнал. 2015; 9 (1): 93-101.

19. Lee $S$, Shi $X Q$, Fan A et al. Targeting macrophage and microglia activation with colony stimulating factor 1 receptor inhibitor is an effective strategy to treat injury-triggered neuropathic pain. Mol Pain. 2018; (14): 1744806918764979. DOI: 10.1177/1744806918764979.

10. Huang $\mathrm{S}$, Frangogiannis NG. Anti-inflammatory therapies in myocardial infarction: failures, hopes and challenges. $\mathrm{Br} J$ Pharmacol. 2018; 175 (9): 1377-400.

11. Itani HA, Harrison DG. Memories that last in hypertension. Am J Physiol Renal Physiol. 2015; 308 (11): F1197-F1199. DOI: 10.1152/ajprenal.00633.2014.

12. Rucker JA, Crowley SD. The role of macrophages in hypertension and its complications Pflugers Arch. 2017; 469 (3-4): 419-30.

13. Hartman MHT, Groot HE, et al. Translational overview of cytokine inhibition in acute myocardial infarction and chronic heart failure. Trends Cardiovasc Med. 2018; 28 (6). DOI: 10.1016/j. tcm.2018.02.003. Available from: https://www.researchgate.net/ publication/260757993_Heart_Failure.

14. Jia D, Cai M, XiY, et al. Interval exercise training increases LIF expression and prevents myocardial infarction-induced skeletal muscle atrophy in rats. Life Sci. 2018; (193): 77-86.

15. Van der Heijden T, Bot I, Kuiper J. The IL-12 cytokine family in cardiovascular diseases. Cytokine. 2017; S1043-4666(17)303150. DOI: 10.1016/j.cyto.2017.10.010.

16. Papageorgiou N, Androulakis E, Papaioannou S, et al. Homoarginine in the shadow of asymmetric dimethylarginine: from nitric oxide to cardiovascular disease. Amino Acids. 2015; 47 (9): 1741-50.

17. Shin S, Thapa SK, Fung H-L. Cellular interactions between L-arginine and asymmetric dimethylarginine: Transport and metabolism. PLoS One. 2017; 12 (5): e0178710. DOI: 10.1371/ journal.pone.0178710.

18. Radaeva OA, Simbirtsev AS. M-CSF, IL-34, VEGF-A kak faktory riska razvitija infarkta miokarda, ostrogo narushenija mozgovogo krovoobrashhenija u bol'nyh jessencial'noj arterial'noj gipertenziej. Rossijskij immunologicheskij zhurnal. 2015; 9 (1): 93-101.

19. Lee $S$, Shi $X Q$, Fan $A$ et al. Targeting macrophage and microglia activation with colony stimulating factor 1 receptor inhibitor is an effective strategy to treat injury-triggered neuropathic pain. Mol Pain. 2018; (14): 1744806918764979. DOI: 10.1177/1744806918764979. 